\title{
UK Renal Registry 19th Annual Report: Appendix K Renal Centre Names and Abbreviations used in the Figures and Data Tables
}

\section{Adult Centres}

\begin{tabular}{|c|c|c|}
\hline City & Hospital & Abbreviation \\
\hline \multicolumn{3}{|l|}{ England } \\
\hline Basildon & Basildon University Hospital & Basldn \\
\hline Birmingham & Heartlands Hospital & B Heart \\
\hline Birmingham & Queen Elizabeth Hospital & B QEH \\
\hline Bradford & St Luke's Hospital & Bradfd \\
\hline Brighton & Royal Sussex County Hospital & Brightn \\
\hline Bristol & Southmead Hospital & Bristol \\
\hline Cambridge & Addenbrooke’s Hospital & Camb \\
\hline Carlisle & Cumberland Infirmary & Carlis \\
\hline Carshalton & St Helier Hospital & Carsh \\
\hline Chelmsford & Broomfield Hospital & Chelms \\
\hline Colchester & Colchester General Hospital & Colchr \\
\hline Coventry & University Hospital Coventry and Warwickshire & Covnt \\
\hline Derby & Royal Derby Hospital & Derby \\
\hline Doncaster & Doncaster Royal Infirmary & Donc \\
\hline Dorset & Dorset County Hospital & Dorset \\
\hline Dudley & Russells Hall Hospital & Dudley \\
\hline Exeter & Royal Devon and Exeter Hospital & Exeter \\
\hline Gloucester & Gloucestershire Royal Hospital & Glouc \\
\hline Hull & Hull Royal Infirmary & Hull \\
\hline Ipswich & Ipswich Hospital & Ipswi \\
\hline Kent & Kent and Canterbury Hospital & Kent \\
\hline Leeds & St James's University Hospital and Leeds General Infirmary & Leeds \\
\hline Leicester & Leicester General Hospital & Leic \\
\hline Liverpool & Aintree University Hospital & Liv Ain \\
\hline Liverpool & Royal Liverpool University Hospital & Liv Roy \\
\hline London & St. Bartholomew's Hospital and The Royal London Hospital & L Barts \\
\hline London & St George's Hospital and Queen Mary's Hospital & L St. G \\
\hline London & Guy's Hospital and St Thomas' Hospital & L Guys \\
\hline London & Hammersmith, Charing Cross, St Mary's & L West \\
\hline London & King's College Hospital & L Kings \\
\hline London & Royal Free, Middlesex and UCL Hospitals & L Rfree \\
\hline Manchester & Manchester Royal Infirmary & M RI \\
\hline Middlesbrough & The James Cook University Hospital & Middlbr \\
\hline Newcastle & Freeman Hospital and Royal Victoria Infirmary & Newc \\
\hline Norwich & Norfolk and Norwich University Hospital & Norwch \\
\hline Nottingham & Nottingham City Hospital & Nottm \\
\hline Oxford & John Radcliffe Hospital and Churchill Hospital & Oxford \\
\hline
\end{tabular}

\section{KARGER}

Fax +4161306 1234 E-Mail karger@karger.com www.karger.com/nef
This article is licensed under the Creative Commons AttributionNonCommercial-NoDerivatives 4.0 International License (CC BYNC-ND) (http:/ ww Us-ND) (htc.//www.karger.com/Services/OpenAccessticense). distribution of modified material requires written permission.
UK Renal Registry, Southmead Hospital, Southmead Road, Bristol, BS10 5NB, UK

Email: renalregistry@renalregistry.nhs.uk 


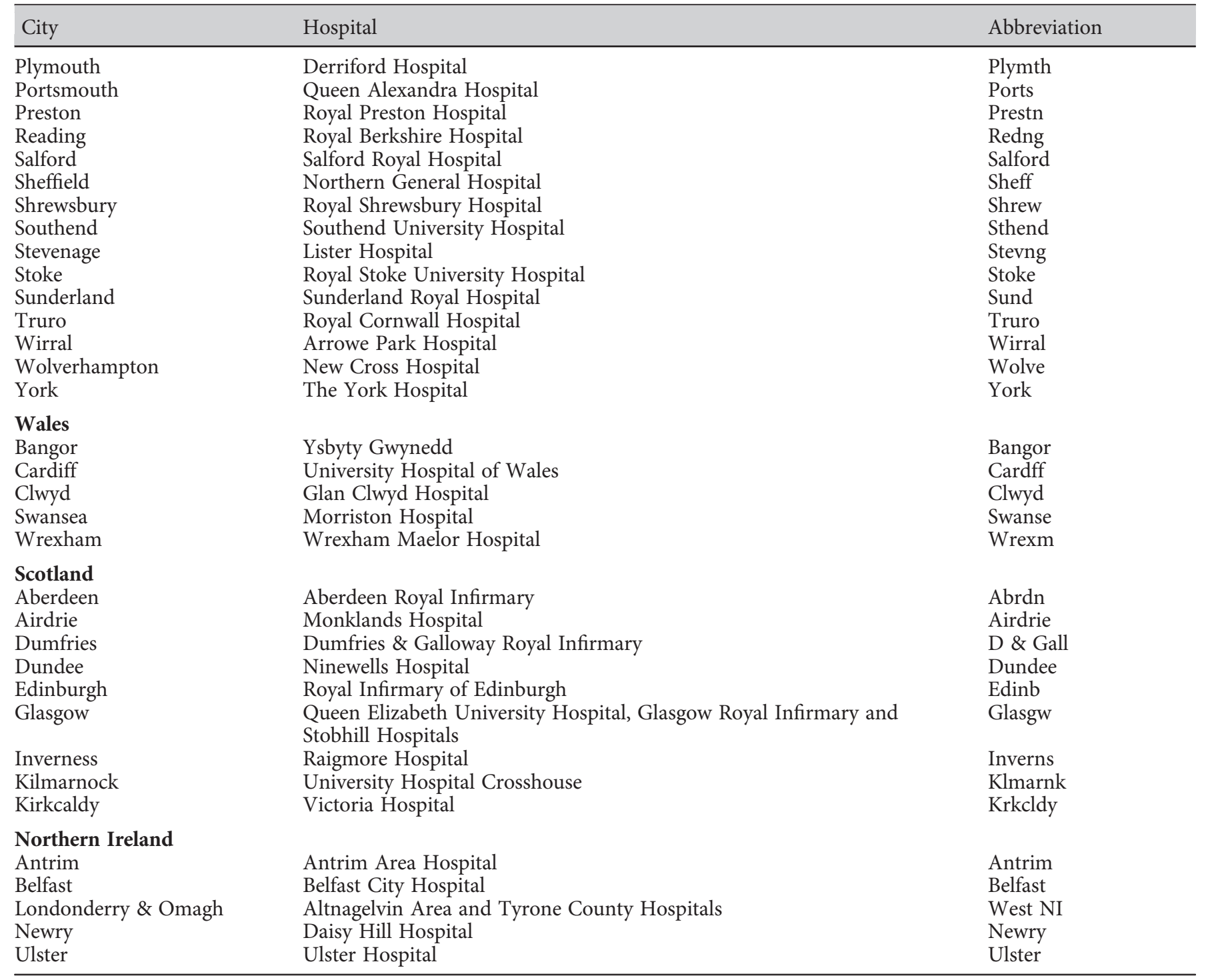

\section{Paediatric Centres}

\begin{tabular}{llll}
\hline City & Hospital & Abbreviation & Country \\
\hline Belfast & Royal Belfast Hospital for Sick Children & Blfst_P & N Ireland \\
Birmingham & Birmingham Children's Hospital & Bham_P & England \\
Bristol & Bristol Royal Hospital for Children & Brstl_P & England \\
Cardiff & KRUF Children's Hospital for Wales & Cardf_P & Wales \\
Glasgow & Royal Hospital for Children & Glasg_P & Scotland \\
Leeds & Leeds Children's Hospital & Leeds_P & England \\
Liverpool & Alder Hey Children's Hospital & Livpl_P & England \\
London & Guy's Hospital - Paediatric & L Eve_P & England \\
London & Great Ormond Street Hospital for Children & Manch_P & England \\
Manchester & Royal Manchester Children's Hospital & Newc_P & England \\
Newcastle & Great North Children's Hospital & Nottm_P & England \\
Nottingham & Nottingham Children's Hospital & England \\
Southampton & Southampton Children's Hospital & & England \\
\hline
\end{tabular}

